\title{
Impact of Current and Power Measurements on Distribution System State Estimation Uncertainty
}

\author{
Marco Pau, Member, IEEE, Paolo Attilio Pegoraro, Member, IEEE, Antonello Monti, Senior Member, IEEE, \\ Carlo Muscas, Senior Member, IEEE, Ferdinanda Ponci, Senior Member, IEEE, Sara Sulis, Member, IEEE
}

\begin{abstract}
The effective management of future smart grids is strictly related to the accurate knowledge of the network operating conditions via Distribution System State Estimation (DSSE). To achieve this target, the measurement infrastructure of the distribution systems needs a substantial upgrade. However, obvious economic limitations prevent a massive deployment of measurement instruments on the field in short times. As a consequence, ad hoc meter placement techniques have to be applied to define location and type of a minimum set of measurements required to obtain the desired accuracy performance. This paper presents a mathematical analysis showing the impact of current and power measurements on the accuracy of DSSE results. The goal is to provide the analytical tools to identify the best placement for additional current or power measurements when applying an incremental deployment of meters in the distribution system. The analysis has a general validity but has also a clear impact on practical situations when the monitoring systems is upgraded starting from an existing infrastructure. Tests on a 95bus sample grid are presented in order to validate the found mathematical results and to highlight the associated benefits in a meter placement perspective.
\end{abstract}

Keywords-Distribution System State Estimation, Weighted Least Squares Method, Uncertainty Analysis, Meter Placement, Current Measurements, Current Estimation.

\section{INTRODUCTION}

Distribution System State Estimation (DSSE) is an essential component of the Distribution Management Systems (DMS) required for the control of future Smart Grids (SGs) [1]. DSSE provides the real-time picture of the operating conditions of the network, which is then used as input by different DMS applications [2]. The accuracy of this information is crucial for the efficient and reliable management of distribution networks (DNs) [3]. To this purpose, a suitable measurement infrastructure is essential to provide the real-time information necessary to the DSSE algorithm to produce accurate results.

However, today's DNs are usually endowed with very few measurement instruments, which often are only available at

M. Pau, A. Monti, F. Ponci are with the Institute for Automation of Complex Power Systems, RWTH Aachen University, Aachen 52062, Germany (email: [mpau, fponci, amonti]@eonerc.rwth-aachen.de)

P. A. Pegoraro, C. Muscas, S. Sulis are with the Department of Electrical and Electronic Engineering of the University of Cagliari, Piazza d'Armi, 09123 Cagliari, Italy (email: [carlo, paolo.pegoraro, sara.sulis]@diee.unica.it).

(c) 2018 IEEE. Personal use of this material is permitted. Permission from IEEE must be obtained for all other users, including reprinting/republishing this material for advertising or promotional purposes, creating new collective works for resale or redistribution to servers or lists, or reuse of any copyrighted components of this work in other works. DOI:10.1109/TIM.2018.2883844 Publisher version:https://ieeexplore.ieee.org/document/8574940 the primary substation. In the DSSE context, the scarcity of measurement devices is usually faced by considering the socalled pseudo-measurements, namely forecasts derived from historical or statistical information about the power generation or consumption at the nodes [4], [5]. Despite making the network observable, the use of pseudo-measurements usually affects the accuracy of DSSE results because of the high uncertainty associated with the forecast information [6]. For this reason, it is well recognized that the reliable management of future SGs requires an important upgrade of the DN measurement system. Nevertheless, considering the large size of DNs, a massive deployment of measurement devices is not predictable, at least in short times, due to the resulting high costs. Therefore, suitable meter placement strategies have to be designed to apply a smart upgrade, likely in an incremental way, of the measurement infrastructure.

In the literature, the meter placement problem for DNs is generally tackled by applying optimization techniques aimed at achieving the desired DSSE accuracy targets with a minimum number of measurement devices. In [7], the focus is on the voltage magnitude estimation and voltage measurements are placed in the nodes with the highest standard deviation to lower the estimation uncertainty below a given threshold. In [8], the optimization problem is modeled through a mixed integer linear programming formulation where the goal is to minimize the Root Mean Square Errors (RMSEs) of the state estimates. The use of heterogeneous devices with different costs and accuracy characteristics, including last generation Phasor Measurement Units (PMUs), is also investigated in other papers (e.g. [9]-[11]). In [12], the uncertainties in the network parameters are also taken into account in addition to the meters uncertainties to ensure the achievement of the desired accuracy targets. The need for better accuracies in some critical points of the network is instead a main consideration in [13], where an incremental placement is applied to fulfil the accuracy requirements. In [14], the Fisher information matrix is chosen as criterion for the meter placement, and the effects brought by possible network topology changes are also considered in the optimization approach. In [15] a mixedinteger programming approach is used to define an optimal meter placement strategy applicable also to DNs with nonobservable conditions.

All the above proposals, while presenting interesting approaches to address the meter placement problem, do not allow a clear understanding of the impact brought by the deployment of additional measurements. The authors of this paper investigated the sources of uncertainty for the estimation 
of the voltage magnitude profile in [16], highlighting the role of both the voltage measurements and the accuracy in the branch current estimates for the final result. In [17], the mathematical analysis was extended to the case of PMUs, pointing out their impact on the voltage phase-angle estimation and the different contribution of conventional and PMU voltage measurements according to their accuracy level.

In this paper, the main goal is to analyze from a mathematical point of view the impact of flow measurements (current and power measurements) on the accuracy of the branch current estimations. Despite several papers reporting experience-gained information about the local impact of flow measurements (see for example [18]), to the best of author's knowledge, no other works investigated analytically this impact. The analysis and results here presented can serve in a meter placement perspective either for obtaining the desired voltage estimation accuracy through a minimum number of devices, as shown in [16], or for achieving specific accuracy targets in the monitoring of the currents in particular branches of the grid.

The rest of this paper is structured as follows. Section II summarizes the peculiarities of DSSE and presents the formulation adopted as a reference for the subsequent analysis. In Section III, the impact of a measurement system composed of only pseudo-measurements is investigated, in order to give an indication of the accuracy achievable with this basic solution. In Section IV, the impact given by additional current or power measurements is presented. Section V shows the tests carried out to prove the validity of the performed mathematical analysis and presents the inherent benefits of the found relationships in a meter placement perspective. Finally, Section VI provides the concluding remarks.

\section{Distribution System State Estimation}

State Estimation (SE) is a well-know mathematical technique used to estimate the most likely state of an electric grid given a redundant set of measurements. The Weighted Least Squares (WLS) method is the most commonly used approach for dealing with SE [19] and is proved to be a reliable solution also for DSSE [20]. It relies on the following measurement model:

$$
\mathbf{z}=\mathbf{h}(\mathbf{x})+\mathbf{e}
$$

where $\mathbf{z}$ is the vector of input measurements at a given time instant, $\mathbf{x}$ is the chosen vector of state variables at the same instant, $\mathbf{h}(\mathbf{x})$ is the vector of the measurement functions linking the measured quantities to the state vector, and $\mathbf{e}$ is measurement error vector (generally assumed as composed of uncorrelated and zero mean variables). The DSSE thus aims at obtaining a snapshot of the state of the network at the operating conditions corresponding to the measurement time.

Starting from this measurement model, the WLS method minimizes the following objective function:

$$
J(\mathbf{x})=[\mathbf{z}-\mathbf{h}(\mathbf{x})]^{T} \mathbf{W}[\mathbf{z}-\mathbf{h}(\mathbf{x})]
$$

where $\mathbf{W}$ is a weighting matrix, equal to the inverse of the measurement error covariance matrix $\boldsymbol{\Sigma}_{\mathbf{z}}$, used to assign higher weights to the most accurate measurements.
The minimization of the objective function in (2) can be obtained by iteratively applying the Gauss-Newton method, which leads to the solution of the following equation system at each iteration $k$ of the estimation algorithm:

$$
\mathbf{G} \Delta \mathbf{x}_{k}=\mathbf{H}^{T} \mathbf{W}\left[\mathbf{z}-\mathbf{h}\left(\mathbf{x}_{k}\right)\right]
$$

where $\mathbf{G}=\mathbf{H}^{T} \mathbf{W H}$ is the so-called Gain matrix, $\mathbf{H}$ is the Jacobian of the measurement functions $\mathbf{h}(\mathbf{x})$, and $\Delta \mathbf{x}_{k}$ is the vector used to update the state variables at iteration $k$ according to the following:

$$
\mathbf{x}_{k+1}=\mathbf{x}_{k}+\Delta \mathbf{x}_{k}
$$

The iterative process stops when a given convergence criteria is achieved (usually the desired maximum deviation in $\Delta \mathbf{x}_{k}$ ). As known in the literature (see for example [21]), one of the advantages in using the WLS method is the possibility to obtain, as well as the state estimates, also their corresponding uncertainties. The covariance matrix of the state estimates is given by the inverse of the Gain matrix, thus resulting in:

$$
\boldsymbol{\Sigma}_{\mathbf{x}}=\mathbf{G}^{-1}=\left(\mathbf{H}^{T} \mathbf{W H}\right)^{-1}
$$

The relationship in (5) provides the mathematical link to the uncertainty of the state estimates and thus it will be the basis for the following analysis developed in Sections III and IV.

Despite the many differences between DSSE and the classical SE applied to power systems, the WLS approach can be also applied to DSSE. A peculiarity of DSSE is the possibility to use different representations of the state vector $\mathbf{x}$ in (1). While in transmission systems the common assumption is to identify the state vector with the set of voltage magnitudes and phase-angles, in DSSE alternative formulations can be more advantageous. In this work, the DSSE formulations based on branch currents introduced in [22] will be considered as reference for the following mathematical developments. In the following, for the sake of simplicity, the case of an equivalent single-phase model is considered. The reported expressions become thus more immediate and easier to illustrate, but nothing prevents dealing with a three-phase system by applying similar considerations and generalized expressions. According to [22], the state vector can be written as:

$$
\mathbf{x}=\left[I_{1}, \ldots, I_{N_{b r}}, \theta_{1}, \ldots, \theta_{N_{b r}}, V_{r e f}\right]^{T}
$$

or as:

$$
\mathbf{x}=\left[i_{r 1}, \ldots, i_{r N_{b r}}, i_{x 1}, \ldots, i_{x N_{b r}}, V_{r e f}\right]^{T}=\left[\mathbf{i}_{r}, \mathbf{i}_{x}, V_{r e f}\right]^{T}
$$

where $V_{\text {ref }}$ is the voltage magnitude at a given bus (usually the slack bus at the main substation) that is chosen as the reference for the phase angle, the couples $\left(I_{j}, \theta_{j}\right)$ and $\left(i_{r j}, i_{x j}\right)$ represent the current of the $j$-th branch in polar and rectangular coordinates, respectively, $\mathbf{i}_{r}$ and $\mathbf{i}_{x}$ are the real and imaginary parts of the branch current vector, and $N_{b r}$ is the total number of branches in the grid. Such formulations allow directly obtaining the covariance sub-matrix of the branch current estimates through (5). Moreover, it is worth noting that [23] proved that it is possible to define the WLS estimators so that different formulations provide the same estimation 
uncertainties, regardless of the used state vector, when fed with the same measurements. As a result, the mathematical findings obtained in next Sections have general validity for all the WLS estimators, regardless of the state vector formulation used as reference for the discussion. The most appropriate state vector for each case will be thus used.

In the following, for the sake of simplicity, the case of balanced distribution grids will be considered in order to refer to an equivalent single-phase model. However, it would be rather straightforward to extend the analysis to the case of unbalanced three-phase distribution grids.

\section{IMPACT OF PSEUDO-MEASUREMENTS}

Because of the low number of measurement devices installed on the field, the use of pseudo-measurements is often essential to achieve the observability of distribution grids. Together with the zero-injection nodes (buses where the absence of power consumption or generation is a priori known), these inputs provide the information on the power injections for each bus of the grid. They are referred to as pseudo-measurements because their value and associated uncertainty are the result of statistical representations or the elaboration of nonreal-time/historical measurements. This measurement set, in addition to one voltage measurement (usually available at the primary substation), guarantees the network observability. The system obtained in this way has no redundancy and is equivalent to a power flow scenario where the primary substation is considered as a slack bus and all the other nodes are treated as nodes operating with given active $(\mathrm{P})$ and reactive (Q) power consumption/injection values (henceforth referred to as PQ values).

At distribution level, the grids are very often composed of several feeders operated in radial mode (reclosing switches between feeders can exist but they are normally open and closed only in emergency cases to supply part of a faulty line). The analysis of the branch current uncertainties for a DN in radial configuration is thus, in general, the most interesting for practical cases.

Let us consider the measurement configuration previously described, with a voltage measurement at the primary substation and the PQ values at all the other nodes, and let us refer to the state vector in (7). With such a DSSE formulation, the powers are included in the WLS procedure by converting them into equivalent real and imaginary current injections:

$$
i_{\mathrm{inj}, n}^{e q}=\frac{P_{\mathrm{inj}, n}-j Q_{\mathrm{inj}, n}}{v_{n}^{*}}=i_{\mathrm{inj}, r n}^{e q}+j i_{\mathrm{inj}, x n}^{e q}
$$

where $P_{\mathrm{inj}, n}$ and $Q_{\mathrm{inj}, n}$ are the active and reactive power at the node $n, v_{n}^{*}$ is the complex conjugate of the voltage at the same node, $i_{\mathrm{inj}, n}^{e q}$ is the phasor of the equivalent current injection and $i_{\mathrm{inj}, r n}^{e q}$ and $i_{\mathrm{inj}, x n}^{e q}$ are the associated real and imaginary parts, respectively.

As discussed in [24], expressing the quantities in per unit and considering the voltage magnitude almost equal to 1 p.u. (with respect to the rated voltage) and the phase-angle very small (as it usually happens in DNs), the same uncertainties $\sigma_{P_{\mathrm{inj}, n}}$ and $\sigma_{Q_{\mathrm{inj}, n}}$ of the active and reactive power can be

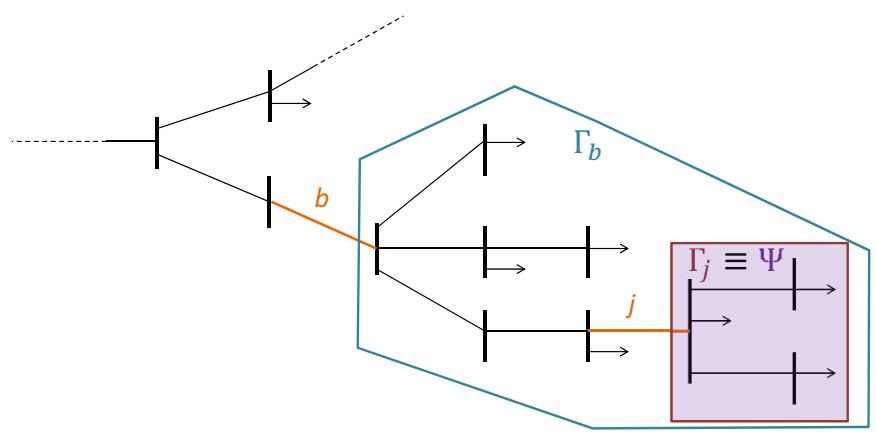

Fig. 1. Nodes downstream branches $b$ and $j$ in a generic network schema.

assigned, in first approximation, to the real and imaginary currents, respectively. Such uncertainties can be thus used to build the weighting matrix. The conversion of the powers in equivalent currents simplifies the definition of the Jacobian matrix $\mathbf{H}_{\text {pse }}$ (where the subscript pse stands for "pseudo" and is used to recall the main information source). As shown in [22] and [24], all the nonzero terms of the Jacobian will be 1 and -1 according to the default direction of the branch currents chosen for the grid. Given this design of the Jacobian $\mathbf{H}_{p s e}$ and the weighting matrix $\mathbf{W}_{p s e}$, the corresponding Gain matrix $\mathbf{G}_{p s e}$ and the covariance matrix of the estimates $\boldsymbol{\Sigma}_{p s e}$ can be computed. From this calculation, the following variances $\Sigma_{p s e, b b}^{\mathbf{i}_{r}}$ and $\Sigma_{p s e, b b}^{\mathbf{i}_{x}}$ can be found for the real and imaginary components of the current in branch $b$, respectively:

$$
\begin{aligned}
\Sigma_{p s e, b b}^{\mathbf{i}_{r}} & =\sum_{h \in \Gamma_{b}} \sigma_{P_{\mathrm{inj}, h}}^{2} \\
\Sigma_{p s e, b b}^{\mathbf{i}_{x}} & =\sum_{h \in \Gamma_{b}} \sigma_{Q_{\mathrm{inj}, h}}^{2}
\end{aligned}
$$

where $\Gamma_{b}$ is the set of all the nodes downstream the branch $b$ (see Fig. 1 for a visual representation of the node sets and branches). These relationships highlight that, with the considered measurement scenario, the branch current uncertainties are given (approximatively) by the quadratic combination of the uncertainties of the current injections downstream the branch, which are assumed to be uncorrelated.

The covariances between the estimates of the currents in branches $j$ and $b$ are instead:

$$
\begin{aligned}
& \Sigma_{p s e, j b}^{\mathbf{i}_{r}}=\sum_{h \in \Psi} \sigma_{P_{\mathrm{inj}, h}}^{2} \\
& \Sigma_{p s e, j b}^{\mathbf{i}_{x}}=\sum_{h \in \Psi} \sigma_{Q_{\mathrm{inj}, h}}^{2}
\end{aligned}
$$

where $\Psi=\left\{\Gamma_{j} \cap \Gamma_{b}\right\}$ is the set of the nodes downstream both branch $j$ and branch $b$ (see Fig. 1). From (11) and (12), it can be found that the covariance is zero when branch $b$ is not downstream branch $j$ (or vice versa), whereas it is equal to the variance of branch $b$ (or $j$ ) if this is downstream branch $j$ (or $b$ ). It is worth noting that in the latter case it is $\Psi=\Gamma_{b}$ (or $\Psi=\Gamma_{j}$ ).

This particular form of the covariance also allows an approximate computation of the correlation factor between the 
current estimates of branches $b$ and $j$. Assuming as an example that branch $b$ is downstream branch $j$, the following holds (calculations are only shown for the real current, but analogous relationships can be derived for the imaginary currents as well):

$$
\Sigma_{p s e, j b}^{\mathbf{i}_{r}}=\Sigma_{p s e, b b}^{\mathbf{i}_{r}}
$$

which leads to the following correlation factor $\rho_{p s e, j b}^{\mathbf{i}_{r}}$ :

$$
\rho_{p s e, j b}^{\mathbf{i}_{r}}=\sqrt{\frac{\boldsymbol{\Sigma}_{p s e, b b}^{\mathbf{i}_{r}}}{\boldsymbol{\Sigma}_{p s e, j j}^{\mathbf{i}_{r}}}}=\sqrt{\frac{\sum_{h \in \Gamma_{b}} \sigma_{P_{\mathrm{inj}, h}}^{2}}{\sum_{h \in \Gamma_{b}} \sigma_{P_{\mathrm{inj}, h}}^{2}+\sum_{k \in \Omega} \sigma_{P_{\mathrm{inj}, k}}^{2}}}
$$

where $\Omega=\Gamma_{j} \backslash \Gamma_{b}$ is the set of nodes between branches $j$ and $b$ (namely, the nodes downstream branch $j$ that are not downstream branch $b$ ). From (14), it is possible to derive the following example correlations:

$$
\rho_{p s e, j b}^{\mathbf{i}_{r}} \begin{cases}\simeq 0 & \text { if } \sum_{h \in \Gamma_{b}} \sigma_{P_{\mathrm{inj}, h}}^{2} \ll \sum_{k \in \Omega} \sigma_{P_{\mathrm{inj}, k}}^{2} \\ \simeq \frac{1}{\sqrt{2}} & \text { if } \sum_{h \in \Gamma_{b}} \sigma_{P_{\mathrm{inj}, h}}^{2} \simeq \sum_{k \in \Omega} \sigma_{P_{\mathrm{inj}, k}}^{2} \\ \simeq 1 & \text { if } \sum_{h \in \Gamma_{b}} \sigma_{P_{\mathrm{inj}, h}}^{2} \gg \sum_{k \in \Omega} \sigma_{P_{\mathrm{inj}, k}}^{2}\end{cases}
$$

In (15), it is possible to observe that the larger the additional uncertainty brought by the current injections in the nodes $k \in$ $\Omega$, the smaller the correlation factor. For the same reason, the closer the branches, the higher the possibility to have high correlation between the current estimates. As it will be shown in Section IV, these elements and values of correlation will play an important role also for determining the effects brought by the placement of additional power/current measurements.

In a meshed grid scenario, an easy analytical derivation of the final current estimate uncertainties cannot be obtained due to the presence of the electrical constraints brought by the Kirchhoff Voltage Law (KVL) along the mesh (see [22] for details on how to integrate them in the branch-current state estimation formulation). In this case, the inverse of the Gain matrix has to be computed to evaluate variances and covariances of each branch current. However, some of the general considerations made for the radial grids still hold. For example, it is easy to predict that the closer the branches, the higher the probability to have large correlations between the current branch estimates. Moreover, current injections with large uncertainty in the nodes between two branches lead to lower correlation between their current estimates.

\section{IMPACT OF BRANCH MEASUREMENTS}

To show analytically the impact brought by additional current measurements, let us indicate with $\mathbf{H}_{A}, \mathbf{W}_{A}$ and $\mathbf{G}_{A}$ the Jacobian, the weighting matrix and the Gain matrix, respectively, associated to a starting measurement configuration available on the field. As indicated in Section II, the corresponding covariance matrix obtainable for the state vector estimations will be $\boldsymbol{\Sigma}_{\mathbf{A}}=\mathbf{G}_{A}^{-1}$. If an additional measurement, independent from those already available, is added to the measurement set, indicating with $\mathbf{H}_{B}$ and $\mathbf{W}_{B}$ the Jacobian and weighting submatrices (which are, respectively, a row vector and a single weight value) related to the new measurement, it is possible to show that:

$$
\begin{array}{r}
\mathbf{G}=\mathbf{H}^{T} \mathbf{W} \mathbf{H}=\left[\begin{array}{ll}
\mathbf{H}_{A}^{T} & \mathbf{H}_{B}^{T}
\end{array}\right]\left[\begin{array}{cc}
\mathbf{W}_{A} & \mathbf{0} \\
\mathbf{0} & \mathbf{W}_{B}
\end{array}\right]\left[\begin{array}{l}
\mathbf{H}_{A} \\
\mathbf{H}_{B}
\end{array}\right] \\
=\mathbf{H}_{A}^{T} \mathbf{W}_{A} \mathbf{H}_{A}+\mathbf{H}_{B}^{T} \mathbf{W}_{B} \mathbf{H}_{B}=\mathbf{G}_{A}+\mathbf{G}_{B}
\end{array}
$$

In the following, a thorough discussion of the impact of different branch measurements is reported.

\section{A. Current Measurements}

Considering an additional branch current measurement, that could be a current magnitude measurement or a synchrophasor measurement from PMU, it is possible to specify (16). Starting with the measurement of the current magnitude, when the state vector is expressed as in (6), the Jacobian matrix $\mathbf{H}_{B}$ is a row vector with only one nonzero entry: a 1 element is present at index $b$ corresponding to the measured current magnitude. The Gain matrix thus becomes:

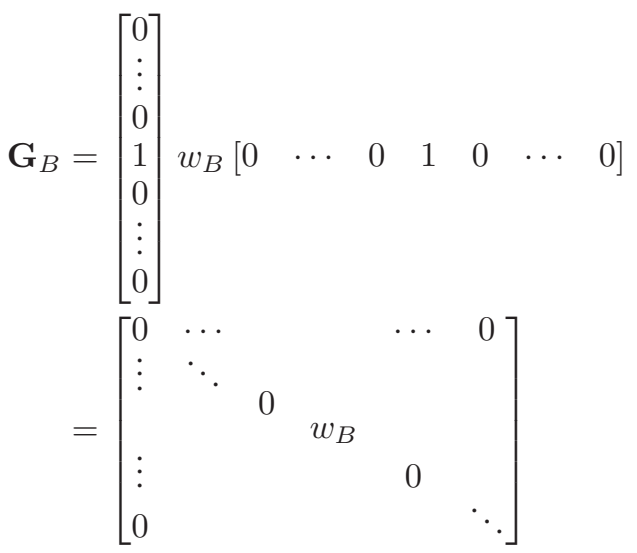

where $w_{B}=\mathbf{W}_{B}$ is the additional measurement weight. The Gain matrix contribution associated to the new measurement at branch $b$ is thus a rank-1 matrix having only one nonzero term equal to the measurement weight on the position $(b, b)$. In this scenario, it is possible to apply the Miller theorem [25] to obtain the overall covariance matrix $\boldsymbol{\Sigma}=\mathbf{G}^{-1}$ and to understand the contribution brought by the additional measurement in the system. According to [25], it is possible to write:

$$
\begin{aligned}
\boldsymbol{\Sigma} & =\left(\mathbf{G}_{A}+\mathbf{G}_{B}\right)^{-1}= \\
& =\mathbf{G}_{A}^{-1}-\frac{1}{1+\operatorname{tr}\left(\mathbf{G}_{B} \mathbf{G}_{A}^{-1}\right)} \mathbf{G}_{A}^{-1} \mathbf{G}_{B} \mathbf{G}_{A}^{-1}
\end{aligned}
$$

where $\operatorname{tr}(\cdot)$ stands for the matrix trace. Considering the particular characteristics of $\mathbf{G}_{B}$ (see (17)), it is possible to find:

$$
\operatorname{tr}\left(\mathbf{G}_{B} \mathbf{G}_{A}^{-1}\right)=\operatorname{tr}\left(\mathbf{G}_{B} \boldsymbol{\Sigma}_{A}\right)=\frac{\sigma_{A b}^{2}}{\sigma_{I_{b}}^{2}}
$$

where $\sigma_{A b}^{2}=\Sigma_{A, b b}$ corresponds to the variance achieved for the current in branch $b$ with the starting measurement configuration and $\sigma_{I_{b}}^{2}=\mathbf{W}_{B}^{-1}$ is the variance associated to the measurement added in this branch. 
Moreover, it is:

$$
\mathbf{G}_{A}^{-1} \mathbf{G}_{B} \mathbf{G}_{A}^{-1}=\frac{1}{\sigma_{I_{b}}^{2}} \boldsymbol{\Lambda}_{v a r}
$$

where $\boldsymbol{\Lambda}_{\text {var }}$ is a positive definite matrix defined by the following generic element:

$$
\boldsymbol{\Lambda}_{v a r, h k}=\boldsymbol{\Sigma}_{A, h b} \boldsymbol{\Sigma}_{A, b k}
$$

Including (19), (20) and (21) in (18), the new estimates covariance matrix becomes:

$$
\boldsymbol{\Sigma}=\boldsymbol{\Sigma}_{A}-\frac{1}{\sigma_{I_{b}}^{2}+\sigma_{A b}^{2}} \boldsymbol{\Lambda}_{v a r}
$$

From (22), it is possible to observe that an additional current measurement clearly leads to an improvement in the currents estimation accuracy, given the negative sign in the second addend of (22). The impact of the new measurement uncertainty appears in the denominator of the negative component of (22): in particular, large uncertainties of the additionally placed measurement lower the achievable improvement by reducing the multiplicative factor for the matrix $\boldsymbol{\Lambda}_{v a r}$. In case $\sigma_{I_{b}}^{2} \ll \sigma_{A b}^{2}$, instead, it is possible to note that the uncertainty of the current estimation in the branch $b$, namely the branch having the new measurement, will become almost equal to the uncertainty of the measurement $I_{b}$ since the covariance term $\Sigma_{b b}=\sigma_{b}^{2}$ will be:

$$
\sigma_{b}^{2}=\frac{\sigma_{A b}^{2} \sigma_{I_{b}}^{2}}{\sigma_{I_{b}}^{2}+\sigma_{A b}^{2}} \simeq \sigma_{I_{b}}^{2}
$$

where the first equality is a weighted average derived from (22) and the second one is the result of the approximation.

For all the other branches of the grid, the resulting improvement in their estimation uncertainty strictly depends on the correlation between the starting estimations in the measured branch $b$ and the considered one $j$ :

$$
\sigma_{j}^{2}=\sigma_{A j}^{2}-\frac{\Sigma_{A, j b}^{2}}{\sigma_{I_{b}}^{2}+\sigma_{A b}^{2}}
$$

As highlighted by eq. (24), the larger the correlation between the current estimations in branches $b$ and $j$, the larger the benefits brought by the additional measurements at branch $b$. The entity of the covariance terms in the starting matrix $\Sigma_{A}$ thus allows quantifying the potential benefits achievable, in terms of currents estimation improvement, through the placement of a current measurement in the generic branch $b$. This result, jointly with the analysis shown in Section III, explains the reason for the local impact of current measurements mentioned in other works: indeed, the closer the branches, the larger the possibility to have strong correlations.

When $\sigma_{I_{b}}^{2} \ll \sigma_{A b}^{2}$, as discussed above, the estimation variance at the generic branch $j$ can be also written as:

$$
\sigma_{j}^{2} \simeq \frac{\sigma_{A j}^{2} \sigma_{I_{b}}^{2}}{\sigma_{A b}^{2}}+\sigma_{A j}^{2}\left(1-\rho_{A, b j}^{2}\right)
$$

where $\rho_{A, b j}$ is the correlation factor. According to the correlation value, the following uncertainties can be derived:

$$
\sigma_{j}^{2} \simeq \begin{cases}\sigma_{A j}^{2} & \text { if } \rho_{A, b j}^{2}=0 \\ \sigma_{A j}^{2}\left(1-\rho_{A, b j}^{2}\right) & \text { if } 0<\rho_{A, b j}^{2}<1 \\ \sigma_{I_{b}}^{2} & \text { if } \rho_{A, b j}^{2}=1\end{cases}
$$

where the last case can be obtained by considering that, if $\sigma_{A j}^{2}$ and $\sigma_{A b}^{2}$ are fully correlated, then it is $\sigma_{A b}^{2}=\sigma_{A j}^{2}$.

Expression (22) also helps understanding the impact of the additional measurement on the new correlation between the estimations of the $b$ th and $j$ th branches. The new correlation coefficient results:

$$
\begin{aligned}
\rho_{j b} & =\frac{\boldsymbol{\Sigma}_{A, j b} \sigma_{I_{b}}^{2}}{\left(\sigma_{I_{b}}^{2}+\sigma_{A b}^{2}\right) \sigma_{b} \sigma_{j}} \\
& =\rho_{A, b j} \frac{\sigma_{A j} \sigma_{I_{b}}}{\sqrt{\sigma_{A j}^{2} \sigma_{I_{b}}^{2}+\sigma_{A j}^{2} \sigma_{A b}^{2}\left(1-\rho_{A, b j}^{2}\right)}} \\
& =\rho_{A, b j} \frac{1}{\sqrt{1+\frac{\sigma_{A b}^{2}}{\sigma_{I_{b}}^{2}}\left(1-\rho_{A, b j}^{2}\right)}}<\rho_{A, b j}
\end{aligned}
$$

where the last inequality holds when the correlation is not complete and becomes ' $\ll$ ' if the measurement uncertainty is much lower, as assumed above. Equation (27) guarantees, when a measurement is added in branch $b$, a decrease in the correlation with the estimations of other branch-currents, thus reducing the beneficial effect brought by further additional current measurements (according to what shown in (24)).

When a current phasor measurement from PMU is considered, equations similar to (18)-(24) hold for the additional phase angle measurement. All the same relationships can be repeated, with the only modification to consider the position $b+N_{b r}$ for the non-zero element in the Jacobian, given the positioning of the current phase-angles in the state vector (6).

\section{B. Power Flow Measurements}

Focusing on power flow measurements, the derivation of the uncertainty reduction due to an additional measurement in branch $b$ follows the same passages as in Section IV-A, since it is possible to translate power measurements into equivalent current ones. It is convenient, in this case, using the state formulation (7), since, for a given branch $b$, an equivalent measurement

$$
i_{b}^{e q}=\frac{P_{b}-j Q_{b}}{v_{n_{b}}^{*}}=i_{r b}^{e q}+j i_{x b}^{e q}
$$

can be defined, where $P_{b}$ and $Q_{b}$ are the active and reactive power measurements at branch $b, v_{n_{b}}^{*}$ is the complex conjugate of the voltage at the node $n_{b}$ of the branch where the power measurement is taken, and subscripts $r$ and $x$ indicate the real and imaginary part of the branch current. As discussed also in Section III, considering small voltage phase angle differences and amplitudes close to 1 p.u., it is: $i_{b}^{e q} \simeq\left(P_{b}-j Q_{b}\right) / V_{n_{b}} \simeq$ $P_{b}-j Q_{b}$. It is thus clear that the description in terms of rectangular coordinates fits perfectly. 
With this assumption, by first adding the $P_{b}$ measurement, equations corresponding to (18)-(22) follow and thus:

$$
\sigma_{b}^{2}=\frac{\sigma_{A b}^{2} \sigma_{i_{r b}^{e q}}^{2}}{\sigma_{i_{r b}^{e q}}^{2}+\sigma_{A b}^{2}} \simeq \sigma_{i_{r b}^{e q}}^{2} \simeq \sigma_{P_{b}}^{2}
$$

where $\sigma_{i_{r b}}^{2}$ and $\sigma_{P_{b}}^{2}$ are the variances of the equivalent and real measurements, respectively, while the first approximation holds when $\sigma_{i_{r b}^{e q}}^{2} \ll \sigma_{A b}^{2}$ and the second one depends on the aforementioned assumptions on the voltages. Analogously:

$$
\sigma_{j}^{2} \simeq \sigma_{A j}^{2}-\frac{\Sigma_{A, j b}^{2}}{\sigma_{P_{b}}^{2}+\sigma_{A b}^{2}}
$$

thus highlighting how, depending on the correlation of the estimations, the new real power flow measurement impacts also on the other branch-current estimations.

Adding the reactive measurements $Q_{b}$ leads to analogous expressions and considerations on the estimation uncertainty, where the starting covariance matrix, the index in the state (now $b+N_{b r}$ ) and the measurement uncertainty are updated accordingly. The reactive power measurement in a given branch directly reduces the uncertainty in the imaginary part of the branch current and affects the other branches to a different extent, depending on the correlation.

\section{Tests And Results}

The analytical findings in Section III and IV are validated in this Section by means of ad hoc simulations. To this purpose, the $11 \mathrm{kV}$ 95-bus grid depicted in Fig. 2 has been used for the tests. The bus numeration is reported in black, while, to avoid confusion, only some of the branch indexes are indicated in red and italics, corresponding to main ramifications and to branches relevant for the analysis in the following ${ }^{1}$. The grid has two large generation points (in nodes 28 and 95), both with an injected power equal to $675 \mathrm{~kW}$, and two large loads (in node 3 and 27), with a power consumption equal to $821 \mathrm{~kW}$ and $678 \mathrm{~kW}$, respectively. All the other loads have a consumption lower than $150 \mathrm{~kW}$. The used line parameters and power consumption/generation values are taken from [26].

As first test case, the basic measurement configuration scenario presented in Section III, composed of only one voltage at the slack bus (node 1) and pseudo-measurements at all the load and generation nodes is considered. The accuracies used here are $1 \%$ and $50 \%$ for the voltage measurement and the pseudo-measurements, respectively. Gaussian variables are associated to these maximum deviations by using a coverage factor of 3 (the same assumption will be used in the following also for current and power measurements). It is important to recall that the pseudo-measurements uncertainty reflects the lack of knowledge on the power loads/generators and thus on the actual operating conditions of the network. The uncertainty assumptions for both pseudo and real measurements are made without loss of generality, since different accuracies can be adopted depending on the available information on network

\footnotetext{
${ }^{1}$ As for the numeration of the branches, each branch index is given by the node number of its end node (the largest one), decreased by one.
}

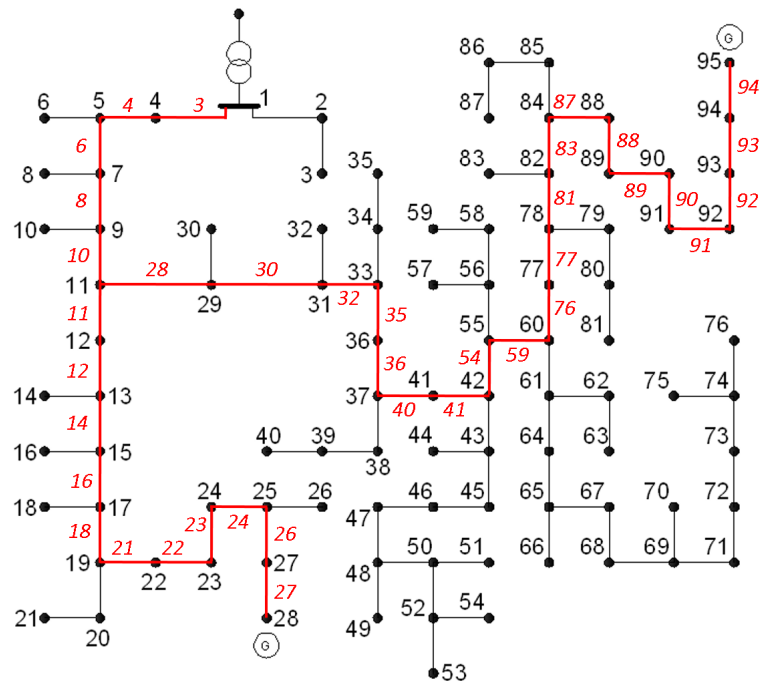

Fig. 2. 95-bus test system.

conditions and knowledge on instrument specifications. Fig. 3 shows the levels of estimation uncertainty (here and in the following, results will refer to the values of expanded uncertainty with coverage factor equal to 3 ) achieved through the inverse of the Gain matrix (5) for the real components of current in the branches between node 1 and node 95 . The reported results obtained from the analytical formulas have been validated also by means of Monte Carlo (MC) simulations. The repeated random trials, corresponding to different extractions of the measurement/pseudo-measurement errors, allow computing the covariance matrix numerically, thus confirming the results.

Coherently with Section III and the analytical expression in (9), it is possible to proceed backwards (bottom-up in the network) and see that the levels of current estimation uncertainty are determined by the incremental contributions of the pseudo-measurement uncertainties. In branch 94, due to the large power generation in node 95 , the uncertainty level is quite large; moving backwards, the additional contribution of uncertainty coming from the other power injections measurements are comparatively small and, therefore, only a slight increment of the uncertainty can be observed. This slight increase is kept till branch 10: here, the current estimation uncertainty also includes the contributions of the pseudo-measurements for all the nodes downstream branch 11. Due to the high power associated to the load in bus 27 and the generator in bus 28 , such contribution is also large and it consequently leads to an evident increase in the estimation uncertainty.

In a similar way, Fig. 4 shows the uncertainty levels for the current estimations (real parts) in the branches between node 1 and node 28. Following the same reasoning, it can be observed that a quite large uncertainty is present in branch 27 due to the large generation at bus 28 . Such an uncertainty steps-up moving to branch 26 , due to the additional contribution brought by the pseudo-measurement associated to the large load at 


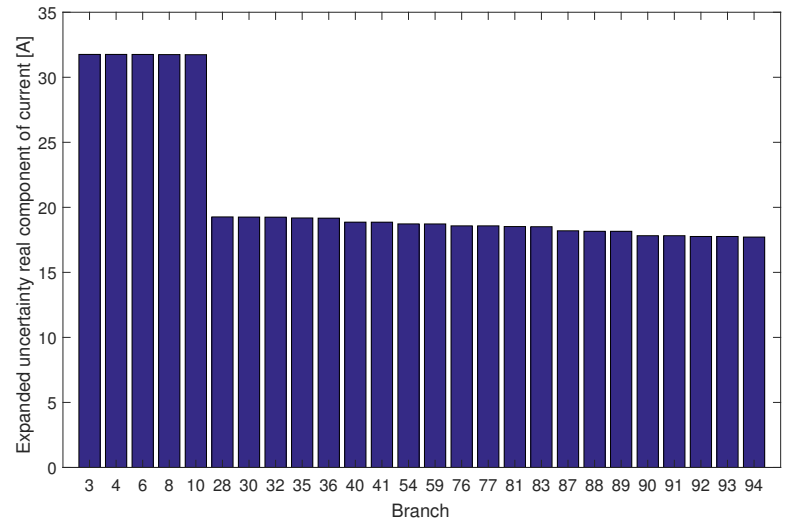

Fig. 3. Uncertainty profile of the real part of current along the path between node 1 and node 95 .

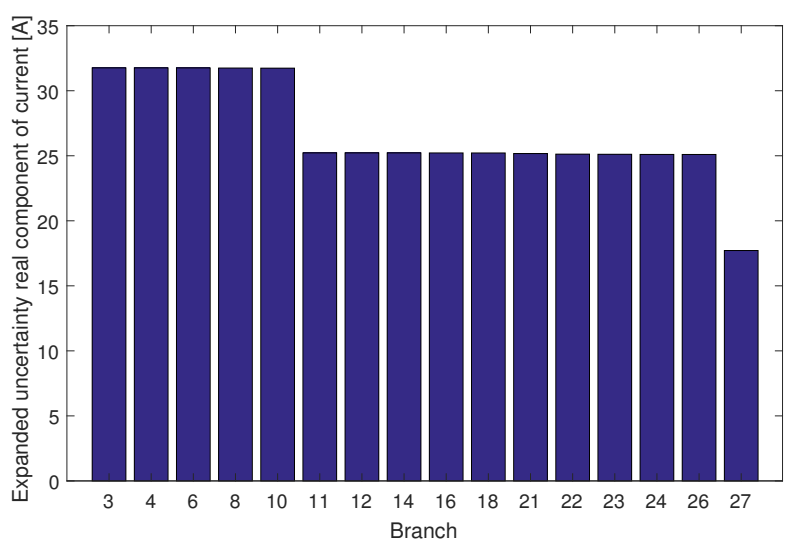

Fig. 4. Uncertainty profile of the real part of current along the path between node 1 and node 28 .

bus 27. Proceeding backwards, the following contributions of uncertainty are relatively smaller and thus produce only a slight increase of current estimation uncertainty. Then, arriving at branch 10, the uncertainty level shows again an important step-up due to the large uncertainty contribution coming from the nodes downstream branch 28 . The results presented in Figs. 3 and 4, which are discussed here mainly from a qualitative viewpoint, have been also evaluated numerically by MC repeated trials, confirming the validity of (9) and (10).

Using the same test scenario, it is possible also to evaluate the covariances and the correlation factors among the current estimations in different branches. Fig. 5 shows the correlation factors for the current estimations (real parts) in the branches between node 1 and 28 with respect to the current estimates in branch 27 and 26. Referring also to (14), it is possible to evaluate the effects brought by the large uncertainties in the pseudo-measurements of large loads or generators. In general, they help increasing the correlation coefficient between two current estimates when they are common to the subtrees of both the considered branches, while they decrease the

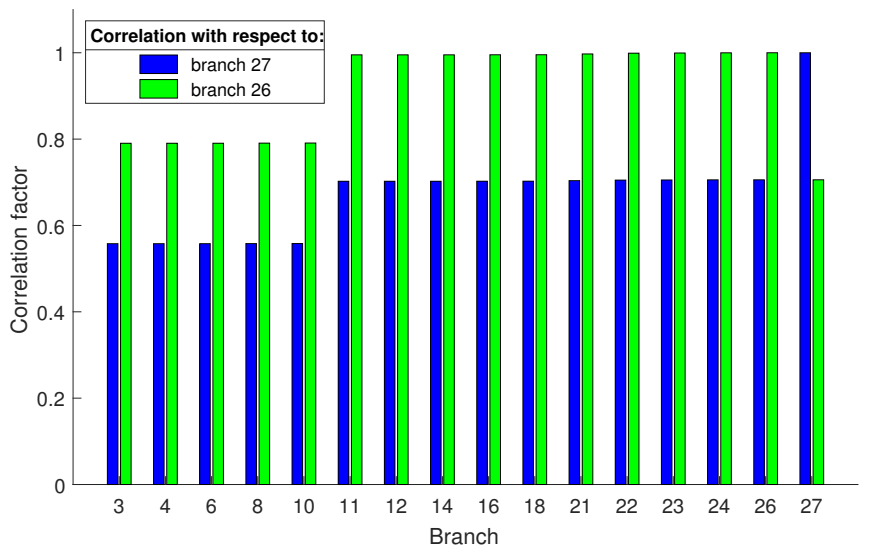

Fig. 5. Trend of the correlation factors for different branch current estimates with respect to the estimations in branch 26 and 27.

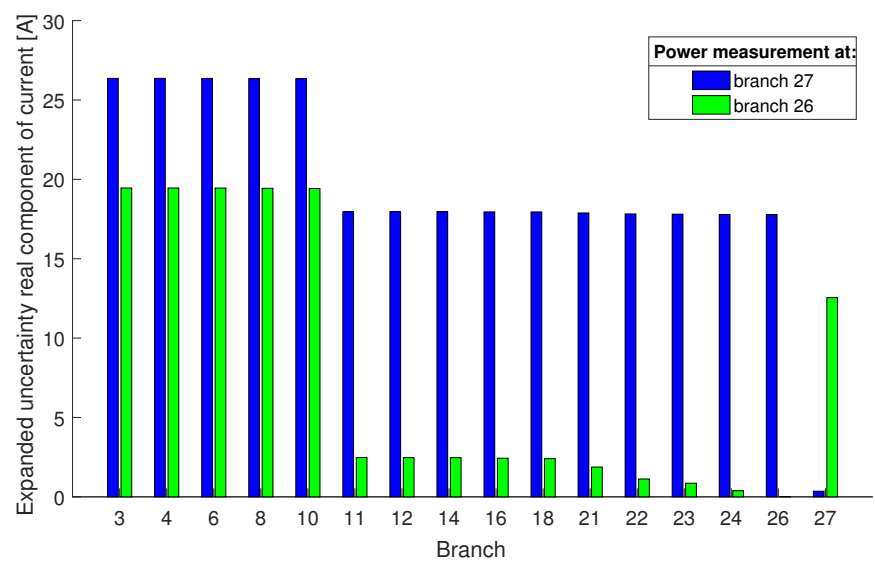

Fig. 6. Impact on the current estimation uncertainty of a different placement of the power measurement.

correlation level when they belong to the set of nodes that is downstream to only one of the branches. This is clearly visible in Fig. 5: the current estimation in branch 26 has a very large correlation with all the estimations in the upstream branches, since the pseudo-measurements with large uncertainty in nodes 27 and 28 are both subtended to the considered branches. On the other hand, the current estimation in branch 27 has a clearly lower correlation factor with the current estimations in the upstream branches. In fact, the large uncertainty contribution given by the pseudo-measurement in node 27 is not subtended by the branch 27 subtree and thus its contribution leads to decorrelation among current estimations.

As discussed in Section IV (and specified in (24)), these correlation factors play a key role to determine the impact of a branch measurement (current or power) on the current estimation uncertainty of the other branches. To show this effect, tests have been carried out by considering a power measurement placed either at branch 27 or at branch 26 (the accuracy of both active and reactive power has been considered equal to $1 \%$ of the actual power value). Fig. 6 highlights 
the different impact resulting from the choice of the power measurement placement. As expected, since in the starting scenario the correlations with the branch current estimate in branch 26 were much higher, this placement leads to evident advantages. In particular, since the correlation factor was almost 1 for the feeder branches from 11 to 24, the uncertainty level is extremely low in those branches and a reduction higher than $90 \%$ is achieved with respect to the uncertainty present in the starting scenario. Such a result highlights how the analytical findings of Section IV can be exploited in a meter placement perspective to identify the locations where the installation of a branch measurement could bring the largest benefits. The analysis of the correlation factors and the performed tests also highlight how the additional branch measurement has a local impact and only affects the current estimates of a subset of branches of the grid. Coherently with (24), indeed, only the upstream branches (between node 1 and the measured branch) and the downstream branches have an improved current estimation. For all the other branches (those not shown in Fig. 6), since the correlation with the current estimates in branch 26 and 27 is zero, no estimation improvement is obtained.

The effects of the correlation factors on the potential improvements for the branch current estimation have been also evaluated in a quantitative way, to assess the correctness of the relationships presented in Section IV. Tables I and II provide some examples of the obtained results, referring to the placement of a power measurement at branch 87 and a current magnitude measurement at branch 10 , respectively. In these tests, both the measurements have been considered to have an accuracy equal to $1 \%$. Tables I and II show, once again, how significant improvements can be achieved on the estimation of those branch currents that are highly correlated with the current estimation of the measured branch. Moreover, it can be noticed that the results achieved with the analytical expressions given in (30) and (24) for the computation of the refinement of the current estimation uncertainty (for the additional power and current magnitude measurement, respectively) are identical or very close to the results achievable via MC simulation. This proves the validity of the mathematical relationships derived in Section IV. These relationships can be thus used, in a meter placement perspective, as a basis to estimate the uncertainty improvements achievable through an incremental deployment of branch current/power meters and to figure out the best locations for the installation of new measurement devices. This can be important to achieve desired accuracy targets on the knowledge of the current/power flow in specific branches of the grid, but also for the accurate estimation of the voltage profiles, since, as demonstrated in [16] and [17], the current flows and the associated voltage drops play a crucial role for the accuracy of the voltage estimations.

The last columns of Tables I and II provide instead the view on the updated values of correlation (in terms of correlation coefficients after the measurement placement) between the current estimations in the considered branch and the branch with the measurement. Coherently with (27), it can be observed that a much smaller value of correlation is obtained. Only in Table II, it can be noticed that a still important correlation,
TABLE I. IMPACT OF ACTIVE POWER MEASUREMENT AT BRANCH 87 ON THE REAL CURRENT ESTIMATES

\begin{tabular}{|c|c|c|c|c|c|}
\hline \multirow{2}{*}{ Branch } & \multirow{2}{*}{$\begin{array}{c}\text { Starting } \\
\text { uncertainty } \\
{[\mathrm{A}]}\end{array}$} & \multirow{2}{*}{$\begin{array}{c}\text { Correlation } \\
\text { with meas. } \\
\text { branch }\end{array}$} & \multicolumn{2}{|c|}{ New uncertainty $[A]$} & \multirow{2}{*}{$\begin{array}{c}\text { New corr. } \\
\text { coeff. } \\
\text { with meas. } \\
\text { branch }\end{array}$} \\
\hline & & & Theoretical & Simulation & \\
\hline 8 & 31.75 & 0.57 & 26.01 & 26.01 & 0.01 \\
\hline 16 & 25.22 & 0 & 25.22 & 25.22 & 0 \\
\hline 30 & 19.25 & 0.95 & 6.29 & 6.29 & 0.03 \\
\hline 94 & 17.71 & 0.97 & 4.06 & 4.06 & 0.05 \\
\hline
\end{tabular}

TABLE II. IMPACT OF CURRENT MAGNITUDE MEASUREMENT AT BRANCH 10 ON THE CURRENT MAGNITUDE ESTIMATES

\begin{tabular}{|c|c|c|c|c|c|}
\hline \multirow{2}{*}{ Branch } & \multirow{2}{*}{$\begin{array}{c}\text { Starting } \\
\text { uncertainty } \\
{[\mathrm{A}]}\end{array}$} & \multirow{2}{*}{$\begin{array}{c}\text { Correlation } \\
\text { with meas. } \\
\text { branch }\end{array}$} & \multicolumn{2}{|c|}{ New uncertainty $[\mathbf{A}]$} & \multirow{2}{*}{$\begin{array}{c}\text { New corr. } \\
\text { coeff. } \\
\text { with meas. } \\
\text { branch }\end{array}$} \\
\hline & & & Theoretical & Simulation & \\
\hline 8 & 22.90 & 0.99 & 0.88 & 0.88 & 0.79 \\
\hline 16 & 10.94 & 0.71 & 7.66 & 7.55 & 0.03 \\
\hline 30 & 16.00 & 0.60 & 12.79 & 12.76 & 0.02 \\
\hline 94 & 16.57 & -0.49 & 14.39 & 14.42 & -0.02 \\
\hline
\end{tabular}

even if lower, is present between the estimates in branch 8 and 10 (these branches are adjacent). Such a result suggests, for example, that the placement of an additional power/current measurement on the same branch would not bring almost any improvement for the current estimation on the other branches.

Additional tests are finally presented to show how the analytical findings could be exploited in a simple way to derive possible meter placement criteria. Starting from the basic measurement configuration with only a voltage measurement at the main substation, it is assumed, as an example, that three additional measurement points, each including one voltage and one PQ power measurement, are placed to improve the voltage estimation profile. In [16] and [17] it was proved that solutions relying only upon the use of voltage measurements are inefficient since they neglect the impact on the voltage uncertainty brought by the currents (and the related voltage drops), thus leading to a larger number of measurement points.

Based on this and following a criterion often used in the literature, a strategy could be devised for an incremental deployment of the meters ("Placement A" in the following), which identifies at each step the bus with highest voltage uncertainty and allocates a voltage measurement on that bus and a power flow measurement on the arriving branch. A second strategy ("Placement B"), which considers instead the analytical findings of this paper, could be to identify the branch with the highest correlations to the current estimation of the other branches in the grid and use it and its end node for the power and voltage measurements, respectively. This can be done for example by finding, at each placement step, the branch having the largest average correlation factor with the other branches.

Table III shows the final locations of the voltage and power measurements when applying the above strategies to place the additional measurement points (it is worth noticing that each pair of voltage and PQ measurements can be installed at the 
TABLE III. LOCATION OF THE ADDITIONAL MEASUREMENTS FOR DIFFERENT PLACEMENT STRATEGIES

\begin{tabular}{|c|c|c|}
\hline $\begin{array}{c}\text { Placement } \\
\text { strategy }\end{array}$ & $\begin{array}{c}\text { Bus voltage } \\
\text { measurements }\end{array}$ & $\begin{array}{c}\text { Branch power } \\
\text { measurements }\end{array}$ \\
\hline \hline Placement A & $27,28,95$ & $26,27,94$ \\
Placement B & $5,19,84$ & $4,18,83$ \\
\hline
\end{tabular}

same bus with a single measurement device). Fig. 7 shows instead the associated profile of expanded uncertainty for the voltage estimation, which is obtained by using both voltage and power measurements with an accuracy equal to $1 \%$. It is possible to observe that, in this scenario, both the strategies give similar results, with Placement B guaranteeing a slightly flatter profile of voltage estimation uncertainty, due to the fact that branches having a larger impact on the current estimation of the remaining branches have been chosen for the placement of the power meters (thus acting on the uncertainties in the voltage drops with a beneficial effect on the overall voltage estimation profile). Different considerations can be drawn when looking at the uncertainties of the current estimations. Fig. 8 shows the current magnitude expanded uncertainty given by the two meter placement solutions for the branches in the feeders highlighted in Fig. 2. It is possible to observe that Placement B allows having a lower estimation uncertainties for more branches (the average expanded uncertainty among all the branches of the grid is $1.84 \mathrm{~A}$, which is $20 \%$ lower than Placement A), even if the expanded uncertainty of branch 27 is much higher because of its low correlation with the other branches. The reported results show that two simple (clearly non-optimal) placement approaches can lead to very different uncertainty profiles for the branch currents, while other complementary criteria could be applied to improve the accuracy and satisfy specific objectives. The design of a detailed meter placement strategy is out of the scope of this paper, but the above results give an idea on how the mathematical findings of this paper could be exploited for the design of efficient and incremental meter placement solutions.

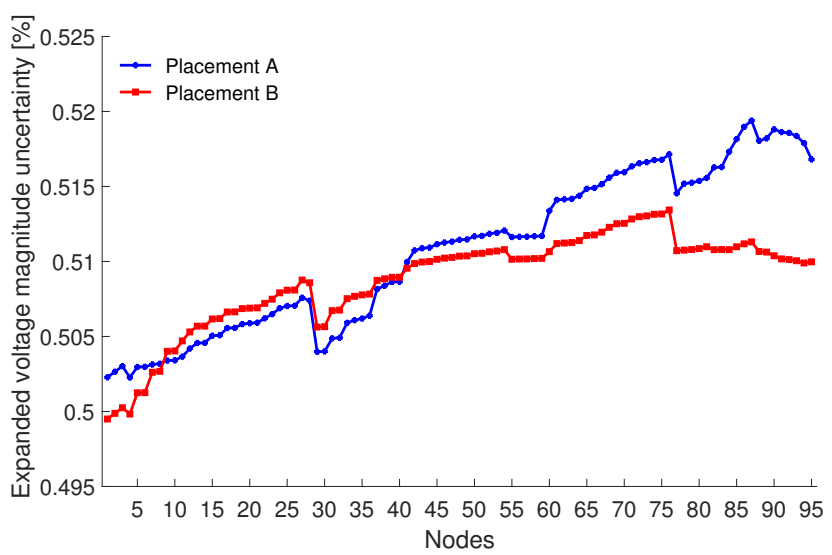

Fig. 7. Voltage estimation uncertainty with different placement strategies.

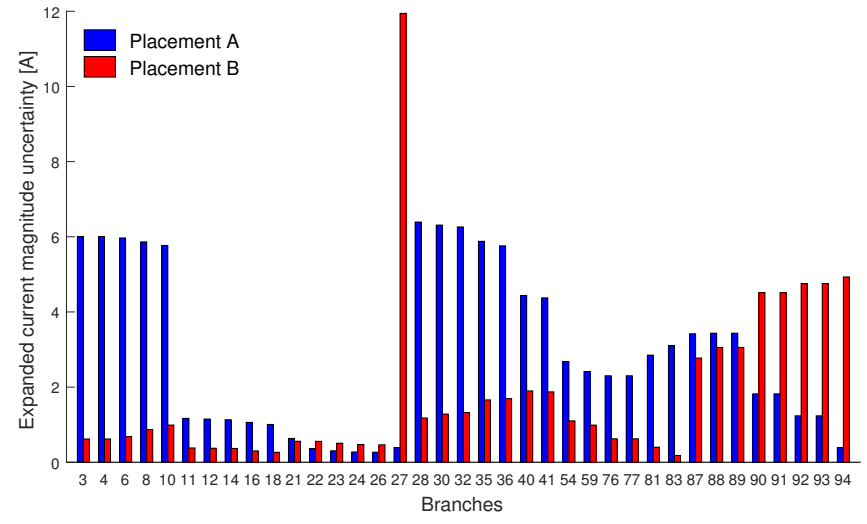

Fig. 8. Current estimation uncertainty in the main feeders with different placement strategies.

\section{CONCLUSIONS}

This paper presented a mathematical analysis aimed at identifying the impact of current and power measurements on the estimation uncertainty of the branch currents in the grid. The derived formulation allows predicting the potential improvements achievable in terms of accuracy of the branch current estimations, through the deployment of power and current measurements, depending on their placement. In this way, it becomes possible to identify the most convenient location for the meter placement, according to the desired accuracy targets. Performed tests show the potential value in a meter placement perspective and prove the validity of the derived expressions. This mathematical framework can serve thus as a useful tool for the design of meter placement strategies aimed at guaranteeing specific accuracy targets with a minimal installation of measurement instruments and thus it can be very helpful in practice when dealing with a monitoring system upgrade.

\section{ACKNOWLEDGMENTS}

This work was partially funded by the Excellence Initiative of the German federal and state governments.

Dr. Pegoraro work was partially funded by Fondazione di Sardegna for the research project "SUM2GRIDS, Solutions by mUltidisciplinary approach for intelligent Monitoring and Management of power distribution GRIDS".

\section{REFERENCES}

[1] J. Fan and S. Borlase, "The evolution of distribution," Power and Energy Magazine, IEEE, vol. 7, no. 2, pp. 63-68, March 2009.

[2] A. Meliopoulos, E. Polymeneas, Z. Tan, R. Huang, and D. Zhao, "Advanced distribution management system," IEEE Trans. Smart Grid, vol. 4, no. 4, pp. 2109-2117, Dec 2013.

[3] G. Celli, P. A. Pegoraro, F. Pilo, G. Pisano, and S. Sulis, "DMS cyberphysical simulation for assessing the impact of state estimation and communication media in smart grid operation," IEEE Transactions on Power Systems, vol. 29, no. 5, pp. 2436-2446, Sept 2014. 
[4] E. Manitsas, R. Singh, B. C. Pal, and G. Strbac, "Distribution system state estimation using an artificial neural network approach for pseudo measurement modeling," IEEE Trans. Power Syst., vol. 27, no. 4, pp. 1888-1896, Nov 2012.

[5] F. Adinolfi, F. D’Agostino, A. Morini, M. Saviozzi, and F. Silvestro, "Pseudo-measurements modeling using neural network and fourier decomposition for distribution state estimation," in IEEE PES Innovative Smart Grid Technologies, Europe, Oct 2014, pp. 1-6.

[6] A. Angioni, T. Schlsser, F. Ponci, and A. Monti, "Impact of pseudomeasurements from new power profiles on state estimation in lowvoltage grids," IEEE Trans. Instrum. Meas., vol. 65, no. 1, pp. 70-77, Jan 2016

[7] A. Shafiu, N. Jenkins, and G. Strbac, "Measurement location for state estimation of distribution networks with generation," Generation, Transmission and Distribution, IEE Proceedings-, vol. 152, no. 2, pp. 240-246, 2005

[8] X. Chen, J. Lin, C. Wan, Y. Song, S. You, Y. Zong, W. Guo, and Y. Li, "Optimal meter placement for distribution network state estimation: A circuit representation based MILP approach," IEEE Trans. Power Syst., vol. 31, no. 6, pp. 4357-4370, Nov 2016

[9] J. Liu, J. Tang, F. Ponci, A. Monti, C. Muscas, and P. Pegoraro, "Tradeoffs in pmu deployment for state estimation in active distribution grids," IEEE Trans. Smart Grid, vol. 3, no. 2, pp. 915-924, 2012.

[10] J. Liu, F. Ponci, A. Monti, C. Muscas, P. Pegoraro, and S. Sulis, "Optimal meter placement for robust measurement systems in active distribution grids," IEEE Trans. Instrum. Meas., vol. 63, no. 5, pp. 1096-1105, May 2014.

[11] S. Prasad and D. M. V. Kumar, "Trade-offs in PMU and IED deployment for active distribution state estimation using multi-objective evolutionary algorithm," IEEE Trans. Instrum. Meas., vol. 67, no. 6, pp. 1298-1307, 2018.

[12] C. Muscas, F. Pilo, G. Pisano, and S. Sulis, "Optimal allocation of multichannel measurement devices for distribution state estimation," IEEE Trans. Instrum. Meas., vol. 58, no. 6, pp. 1929-1937, 2009.

[13] Y. Xiang, P. F. Ribeiro, and J. F. G. Cobben, "Optimization of stateestimator-based operation framework including measurement placement for medium voltage distribution grid," IEEE Trans. Smart Grid, vol. 5, no. 6, pp. 2929-2937, Nov 2014.

[14] M. G. Damavandi, V. Krishnamurthy, and J. R. Mart, "Robust meter placement for state estimation in active distribution systems," IEEE Trans. Smart Grid, vol. 6, no. 4, pp. 1972-1982, July 2015.

[15] T. C. Xygkis and G. N. Korres, "Optimized measurement allocation for power distribution systems using mixed integer SDP," IEEE Trans. Instrum. Meas., vol. 66, no. 11, pp. 2967-2976, Nov 2017.

[16] M. Pau, P. A. Pegoraro, S. Sulis, and C. Muscas, "Uncertainty sources affecting voltage profile in distribution system state estimation," in 2015 IEEE International Instrumentation and Measurement Technology Conference (I2MTC) Proceedings, May 2015, pp. 109-114.

[17] C. Muscas, M. Pau, P. A. Pegoraro, and S. Sulis, "Uncertainty of voltage profile in PMU-based distribution system state estimation," IEEE Trans. Instrum. Meas., vol. 65, no. 5, pp. 988-998, May 2016.

[18] P. Janssen, T. Sezi, and J. Maun, "Meter placement impact on distribution system state estimation," in Electricity Distribution (CIRED 2013), 22nd International Conference and Exhibition on, Jun. 2013, pp. 1-4.

[19] A. Abur and A. G. Expòsito, Power System State Estimation. Theory and Implementation. Marcel Dekker, New York, 2004.

[20] R. Singh, B. C. Pal, and R. A. Jabr, "Choice of estimator for distribution system state estimation," IET Generation, Transmission Distribution, vol. 3, no. 7, pp. 666-678, July 2009.

[21] A. Gomez-Exposito, A. Abur, A. de la Villa Jaen, and C. Gomez-Quiles, "A multilevel state estimation paradigm for smart grids," Proceedings of the IEEE, vol. 99, no. 6, pp. 952-976, June 2011.

[22] M. Pau, P. A. Pegoraro, and S. Sulis, "Efficient branch-current-based distribution system state estimation including synchronized measure- ments," IEEE Trans. Instrum. Meas., vol. 62, no. 9, pp. 2419-2429, Sep. 2013.

[23] M. Pau, P. A. Pegoraro, and S. Sulis, "Performance of three-phase WLS distribution system state estimation approaches," in Applied Measurements for Power Systems (AMPS), 2015 IEEE International Workshop on, Sept 2015, pp. 138-143.

[24] M. Baran and A. Kelley, "A branch-current-based state estimation method for distribution systems," IEEE Trans. Power Syst., vol. 10, no. 1, pp. 483-491, Feb. 1995.

[25] K. S. Miller, "On the inverse of the sum of matrices," Mathematics Magazine, vol. 54, no. 2, pp. 67-72, Mar. 1981. [Online]. Available: http://www.jstor.org/stable/2690437

[26] L. M. P. dos Santos, "Aperfeicoamento de um algoritmo de estimacao de estados para rede de distribuicao considerando grande penetracao de producao dispersa," Master's thesis, Faculdade de Engenharia da Universidade do Porto, Porto, Jun. 2008.

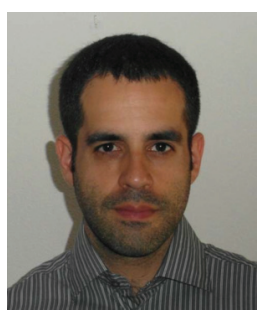

Marco Pau (S'14-M'15) received the M.S. degree (cum laude) in electrical engineering and the Ph.D. degree in electronic engineering and computer science from the University of Cagliari, Italy, in 2011 and 2015, respectively. Currently, he is research associate at the Institute for Automation of Complex Power Systems at the E.ON Energy Research Center, RWTH Aachen University, where he is leading the team for Power System Automation. His research activities mainly concern the design of measurement systems for the monitoring and management of distribution systems the development of solutions for distribution grid automation.

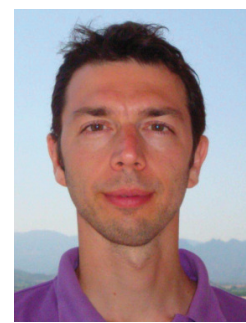

Paolo Attilio Pegoraro (M'06) received the M.S (summa cum laude) degree in telecommunications engineering and the Ph.D. degree in electronic and telecommunications engineering from the University of Padova, Padua, Italy, in 2001 and 2005, respectively. He is currently an Assistant Professor of electrical and electronic measurements with the Department of Electrical and Electronic Engineering, University of Cagliari, Cagliari, Italy. He received the National Scientific Qualification to function as an Associate Professor in 2017. He has authored or co-authored over 80 scientific papers. His current research interests include the development of new architectures and measurement techniques for the monitoring of modern power networks, with particular attention to synchronized measurements and state estimation for distribution grids. Dr. Pegoraro is a member of the IEEE Instrumentation and Measurement Society TC-39 Measurements in Power Systems. 


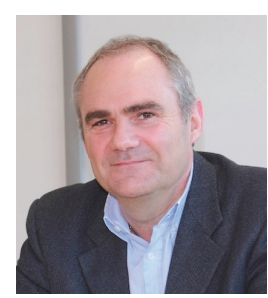

Antonello Monti received his M.Sc degree (summa cum laude) and his $\mathrm{PhD}$ in Electrical Engineering from Politecnico di Milano, Italy in 1989 and 1994 respectively. He started his career in Ansaldo Industria and then moved in 1995 to Politecnico di Milano as Assistant Professor. In 2000 he joined the Department of Electrical Engineering of the University of South Carolina (USA) as Associate and then Full Professor. Since 2008 he is the director of the Institute for Automation of Complex Power System within the E.ON Energy Research Center at RWTH Aachen University. Dr. Monti is author or co-author of more than 300 peerreviewed papers published in international Journals and in the proceedings of International conferences. He is a Senior Member of IEEE, Associate Editor of the IEEE System Journal, Associate Editor of IEEE Electrification Magazine, Member of the Editorial Board of the Elsevier Journal SEGAN and member of the founding board of the Springer Journal Energy Informatics. Dr. Monti is the recipient of the 2017 IEEE Innovation in Societal Infrastructure Award.

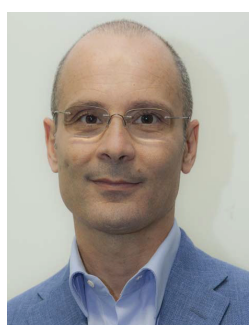

Carlo Muscas (M98, SM15) received the M.S (cum laude) degree in electrical engineering from the University of Cagliari, Cagliari, Italy, in 1994 He was Assistant Professor (from 1996 to 2001) and Associate Professor (from 2001 to 2017) with the University of Cagliari. Since 2017 he has been a Full Professor of Electrical and Electronic Measurement with the University of Cagliari. His current research interests include the measurement of synchronized phasors, the implementation of distributed measurement systems for modern electric grid, and the study of power quality phenomena. He has authored and co-authored more than 140 scientific papers and is currently the Chairman of TC 39 (Measurements in Power Systems) of IEEE Instrumentation and Measurement Society.

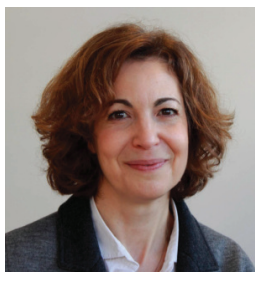

Ferdinanda Ponci graduated with a Ph.D. in Electrical Engineering from Politecnico di Milano (Italy), in 2002. She joined the Department of Electrical Engineering, University of South Carolina, (USA), as an Assistant Professor in 2003, and became Associate Professor in 2008. In 2009, she joined the Institute for Automation of Complex Power Systems, E.ON Research Center, RWTH Aachen University, Aachen, Germany, where she is currently a Professor for Monitoring and distributed control for power systems. She is currently engaged in research on automation and advanced monitoring of active distribution systems.

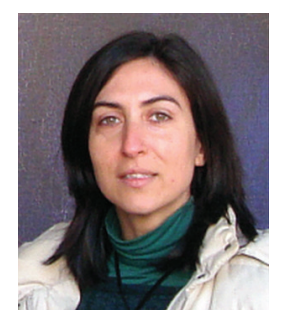

Sara Sulis (M'06) received the M.S. degree in electrical engineering and the Ph.D. degree in industrial engineering from the University of Cagliari, Cagliari, Italy, in 2002 and 2006, respectively. In 2013, she received the National Scientific Qualification to function as an Associate Professor. She is currently an Assistant Professor of Electrical and Electronic Measurements with the University of Cagliari. She has authored or co-authored more than 80 scientific papers. Her current research interests include distributed measurement systems designed to perform state estimation and harmonic sources estimation of distribution networks. Dr. Sulis is currently a member of the IEEE Instrumentation and Measurement Society and the TC-39-Measurements in Power Systems. 